\title{
UNCERTAINTY QUANTIFICATION IN SYNTHETIC APERTURE RADAR REMOTE SENSING DATA PROCESSING
}

\author{
Salvatore Savastano, Raffaella Guida
}

\author{
Surrey Space Centre \\ Faculty of Engineering and Physical Sciences (FEPS) \\ University of Surrey (UK)
}

\begin{abstract}
A new methodology, based on the International Organization for Standardization (ISO) Guide to the expression of Uncertainty in Measurement (GUM), for the analysis of uncertainties in Synthetic Aperture Radar (SAR) remote sensing data is presented. The principal idea is to assess the sources of uncertainty, developing computational approaches to propagate uncertainties through the whole SAR signal processing chain. The final goal is to construct uncertainties budgets to quantify the total uncertainty of SAR products, permitting their 'traceability' to international reference standards.
\end{abstract}

Index Terms - SAR, GUM, uncertainty.

\section{INTRODUCTION}

Synthetic Aperture Radar (SAR) signal processing from airborne and satellite platforms, capable of millimetric resolution, is a modern remote sensing methodology. It is able to generate high resolution images in all weather and light conditions and the final product is comparable with, and complementary to, that obtained with optical sensors. The field of SAR applications is very wide and has grown enormously in recent years: topography, oceanography, geology, monitoring of glacier and snow wetness in glaciology, cropping classification in agriculture, forestry, environment and disaster monitoring represent only some areas where this technology is used [1]. In recent years, the number of SAR sensors in orbit and the quantity of available Earth Observation (EO) data has increased noticeably, pushing the scientists to investigate a way to connect and share all these data and to provide a reliable method to compare different data streams. Moreover, looking at the recommendation made by the Committee on Earth Observation Satellites (CEOS) in 2001 [2], it is also indispensable to ensure that all measurements and associated instrumentation used for any quantitative purpose in remote sensing be fully traceable to International System of Units (SI) as part of the Quality Assurance process. To realise a traceable and trustworthy measurement, robust assessments of the uncertainties associated with SAR products are required. In the early 90 s the metrology community established the GUM [3], a methodology which permits to evaluate, quantify and express uncertainty in any type of measurement. This methodology is widely adopted by many organizations for different scientific activities and it is evident that EO community could not be an exception. Unfortunately the application of GUM is not so straightforward, because the standards provided by this methodology are not consistently implemented in the remote sensing community, where a traditional error analysis framework persists [4]. There have been many achievements in recent years, applying the GUM to optical sensors [5], but there are no other similar studies for the SAR data. So, in this paper for the first time this methodology is applied to evaluate the uncertainties in some steps of the processing chain of SAR data, extending to it the concept of traceability to SI.

The paper is organized as follows. In section 2, a detailed description of the SAR chain processing [6], defining all its component steps and different level-data, is presented. In section 3, the description of GUM is reported, considering all its several tasks, necessary to quantify the uncertainty. In section 4 the first application of the GUM to a simulated scene and the results are provided.

\section{SAR CHAIN OF PROCESSING}

As widely demonstrated in literature, a final SAR image is the result of a complex signal processing. A simplified version of this chain is represented in the flowchart in figure 1. Starting from the EM waves transmitted by the sensor, the signal backscattered from the scene is processed adequately through different steps, permitting to reconstruct the image observed. Through these steps, it is possible to define two levels of SAR data:

- Level-0, also called the RAW data;

- Level-1, also called Single Look Complex (SLC) data.

In this paper the methodology provided by GUM is applied, permitting to identify and to quantify the possible sources of uncertainties along all this chain, relatively to these two types 
of SAR data. As it will be described in section 3, the first stage of GUM is to define the mathematical model which represents these two levels of data and so their detailed description is necessary. For this analysis the stripmap acquisition mode is considered because is the most common geometry for SAR. In this configuration the sensor is mounted on a platform which moves with a constant velocity $v_{p}$, along an ideal straight path at a constant altitude $h$, with no roll, pitch or yaw. In the ideal case the squint angle from broadside to the direction of the beam center is zero and the radar beam is pointed perpendicularly to the flight track, remaining fixed respect to the platform, so that the antenna beam sweeps out a 'strip' on the ground during its movement. The flight direction of the platform is called azimuth or slow time and it is indicated with $y$ or $\eta$, respectively. The direction of the antenna, or line of sight of sensor, is called slant range or fast time and it is indicated with $R$ or $t$, respectively. While moving along its trajectory, the sensor transmits a sequence of closely spaced EM waves at regular intervals, defined by the PRF (Pulse Repetition Frequency) and it records the radar echoes, generated by the Earth's surface. Each point in the scene is illuminated by many pulses (typically by several thousands) because, during this step of transmission/receiving of waves, the platform moves a small distance, occupying different positions along the trajectory. Supposing that the transmitted signal is a 'chirp', the RAW data from a general ground surface, after a quadrature demodulation, which removes the radar carrier frequency, and the sampling in range $\left(F_{r}\right)$, is given by the following convolution [6]:

$$
h_{\text {strip }}(t, \eta)=\gamma(t, \eta) \otimes g_{\text {strip }}(t, \eta)+n(t, \eta)
$$

where

$$
\begin{aligned}
g_{\text {strip }}(t, \eta)= & \operatorname{rect}\left[\frac{(t-2 R(\eta) / c)}{T_{p}}\right] w_{a}(\eta) \\
& \cdot \exp \left[-j 4 \pi f_{c} R(\eta) / c\right] \\
& \cdot \exp \left[j \pi K_{r}(t-2 R(\eta) / c)^{2}\right]
\end{aligned}
$$

is the SAR sensor impulse response, and

$$
R(\eta)=\sqrt{R_{0}^{2}+\left(v_{p} \eta\right)^{2}}
$$

In equations (1)-(3), the following parameters are defined:

- $\gamma(t, \eta)$ is the ground reflectivity.

- $T_{p}$ is the pulse duration time.

- $R(\eta)$ is the slant range from the sensor to a generic point, which varies with azimuth time.

- $R_{0}$ is the minimum range distance between the sensor and the target.

- $c$ is the speed of light.

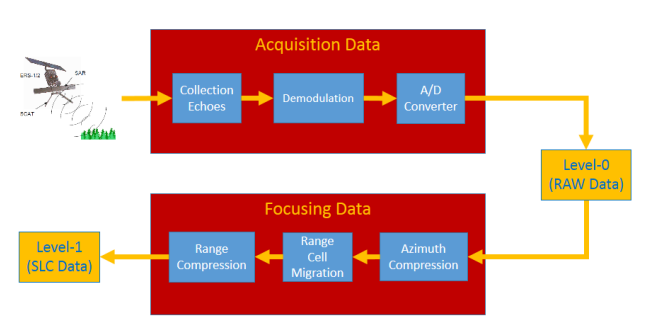

Fig. 1. SAR processing chain

- $w_{a}$ is the azimuth beam pattern.

- $f_{c}$ and $K_{r}$ are respectively the frequency and the chirp rate of the transmitted signal.

- $n(t, \eta)$ is an additional noise component, always present in all electronic components.

The result of this convolution is a 2-D array of complex quantities and it consists of information from the imaged scene spread over a certain interval. To get something similar to optical image, it is necessary to focus the data compressing that and reducing the spread. In the RAW data two chirps are defined: one in the azimuth direction and the other in the range direction. These chirps can be compressed by one dimensional matched filtering in both directions. However, due to the presence of range migration, this process is not direct but it is necessary another step, which compensates this migration effect in range direction. In fact, the received pulses are shifted in range due to the hyperbolic form of the radartarget distance. There are several algorithms to process the raw data. In this paper the Range-Doppler Algorithm (RDA) is adopted due to low computational complexity [7].

\section{MEASUREMENT UNCERTAINTY EVALUATION}

In each measurement the result is considered complete only when also a statement of its uncertainty is defined. Uncertainty is a quantitative measure, which defines a parameter for the quality of a measurement result, enabling its comparison with other results, references, specifications or standards [8]. Very often uncertainty and error are confused with each other. GUM clarifies these two concepts. Error is the difference between the measured value and the 'true value' of the thing being measured. It cannot be eliminated but it can often be reduced. Uncertainty, conversely, takes the form of a range or an interval of an infinite number of values dispersed about the result and it is the quantification of the doubt about the validity of the result of a measurement [3]. The term uncertainty evaluation denotes the procedure that permits to evaluate and to quantify the uncertainty of a measurement. The GUM defines a set of clauses and specific guidelines which establish the correct method to apply for this evaluation. This process is composed by three stages: 
I Formulation, where the scalar output quantity $Y$, the $\mathrm{N}$ input quantities $\mathbf{X}=\left(X_{1}, \ldots, X_{N}\right)^{\top}$ and the measurement function $f$, which links $Y$ and $\mathbf{X}$, are defined. The input quantities represent all possible uncertainty sources for the analysed measurement model and they are statistically described by their Probability Density Functions (PDFs).

II Propagation, where the information, provided by the previous step, is used to determine the PDF for the output quantity $Y$, also regarded as a random variable, propagating the PDFs of the components of $\mathbf{X}$ through the model.

III Summarizing, where the PDF for the output quantity $Y$ is used to get the interval defining the uncertainty associated to the measurement.

There are different approaches to deal with this type of evaluation. In this paper a numerical one, as Monte Carlo method (MCM) is proposed [9]. The MCM considers a small number of steps repeated many times, where each repetition represents a single trial, and the results obtained are aggregated. The method makes repeated random draws from the PDFs describing the input quantities. Given the measurement function and the PDFs for its input quantities, the MCM permits to approximate the PDF for the scalar output $Y$.

\section{APPLICATION OF GUM TO A SIMULATED SCENE}

The main goal of this new methodology is to quantify the uncertainties of the amplitude values pixel by pixel, relatively to Level-0 and Level-1 of a simulated SAR image, starting from statistical distribution of the input parameters. The simulated area (fig. 2) is composed by $N_{r g} \times N_{a z}$ pixels and each of them represents an area whose dimensions are given by the resolution in range and azimuth direction. For the sake of simplicity, in the scene there is only one pixel target, placed in the centre of the area, which reflects back to the sensor most of the received energy, while the background is highly absorbing. This assumption permits to focus the GUM only to this pixel but naturally it is possible to extend this methodology also to a more complex scene. The simulation parameters are reported in tab. 1 and they are based on the ancillary data of ERS-2. For this type of sensor the effect of range migration is negligible and so it is possible not to consider the range correction in the focusing step. As said previously, the first step in an analysis of uncertainty evaluation is to find the mathematical model to link the output and input quantities. Two algorithms in MATLAB language program for RAW and SLC data have been implemented to process the simulated scene and to evaluate the magnitude of the pixels relatively to level0 and level-1. Considering the nominal parameters in tab. 1, the results of the processing chain are presented in fig. 3 and they are consistent with what is widely available in literature.

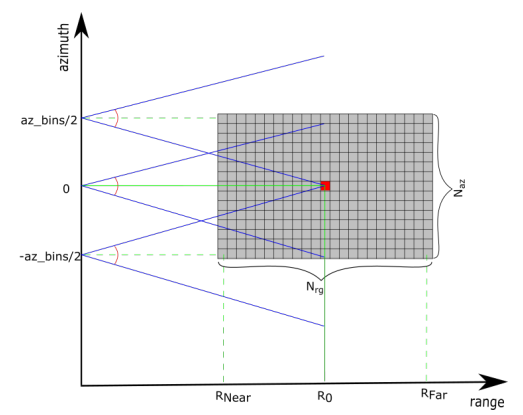

Fig. 2. Imaging geometry of simulated area

The next step in GUM is the description of the input parameters indicated in tab. 1 through suitable PDFs. In this way, the input parameters are not constant anymore but it is possible to do a random draw from their PDFs. These variable parameters represent a new input and they are used to evaluate a new magnitude of the pixel at the centre of the scene for both SAR levels. This procedure is repeated iteratively for many times and aggregating all outputs it is possible to define the Distribution Function (DF) and the PDF of the magnitude of the pixel for level-0 and level-1. An example of this methodology is reported in figs. 4 and 5. In this case all inputs are considered constant and are taken from tab. 1, except the additive noise defined in eq. 1, which is supposed to be described by Gaussian distribution with zero mean and a defined standard deviation. From this PDF $M$-values are taken representing the variable input for the code and $\mathrm{M}$ outputs are evaluated for level-0 and level-1. Aggregating all these values for each level, it is possible to define the DF and the PDF of the output quantity. From these distributions the estimated value $y$ of the magnitude of pixel (assumed as mean), its uncertainty $u(y)$ (assumed as standard deviation) and the endpoints of its coverage interval $y_{L}$ and $y_{H}$ for a given confidence level are evaluated. In the figures it is also reported how well the PDF of the output quantity fits a theoretical Gaussian distribution characterized by the evaluated mean and standard deviation. All results are summarized in tab. 2, named also the Budget Uncertainty. Currently, the statistical description of other input parameters is under investigation and the results will be presented and discussed at the conference.

\begin{tabular}{||c|c||}
\hline$R_{\text {Near }}$ & $852 \mathrm{~km}$ \\
\hline$T_{p}$ & $3.712 \times 10^{-5} \mathrm{~s}$ \\
\hline$K_{r}$ & $4.1899 \times 10^{11} \mathrm{~Hz} / \mathrm{s}$ \\
\hline$F_{r}$ & $18.96 \mathrm{MHz}$ \\
\hline$v_{p}$ & $7098 \mathrm{~m} / \mathrm{s}$ \\
\hline$f_{c}$ & $5.3 \mathrm{GHz}$ \\
\hline$h$ & $690 \mathrm{~km}$ \\
\hline$P R F$ & $1679.9 \mathrm{~Hz}$ \\
\hline$n(t, \eta)$ & 0 \\
\hline
\end{tabular}

Table 1. Input parameters for the simulated scene 


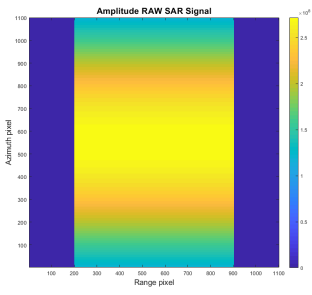

(a) Level-0 data

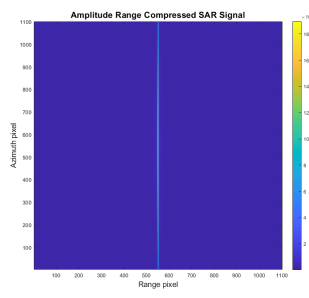

(b) Range compressed data

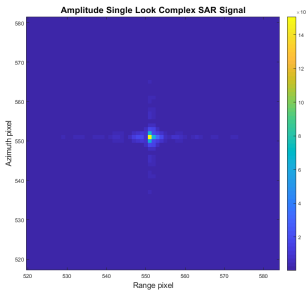

(c) Zoom Level-1 data

Fig. 3. SAR signal proccesing

\begin{tabular}{|c|c|c|c|c|}
\hline \multirow{2}{*}{ Level data } & $\mathbf{y}$ & $\mathbf{u}(\mathbf{y})$ & \multicolumn{2}{|c|}{$\begin{array}{c}\text { Endpoints of 95\% } \\
\text { coverage interval }\end{array}$} \\
\hline RAW & 1.0012 & 0.2002 & 0.6083 & 1.3943 \\
\hline SLC $\left(10^{5}\right)$ & 5.5468 & 0.001186917 & 5.5445 & 5.5491 \\
\hline
\end{tabular}

Table 2. Budget uncertainties for $M=50000$ trials

\section{CONCLUSIONS}

A new methodology for the uncertainty evaluation applied to SAR system has been presented and it represents a complete novelty in the SAR field and more generally in remote sensing community. The description of the SAR processing chain has been introduced. The GUM methodology has been presented and its steps discussed. The mathematical models representing Level-0 and Level-1 SAR data have been implemented in two Matlab codes and a first application of GUM to a simple scenario has been presented. Such methodology in SAR will allow the evaluation of the uncertainties in all different
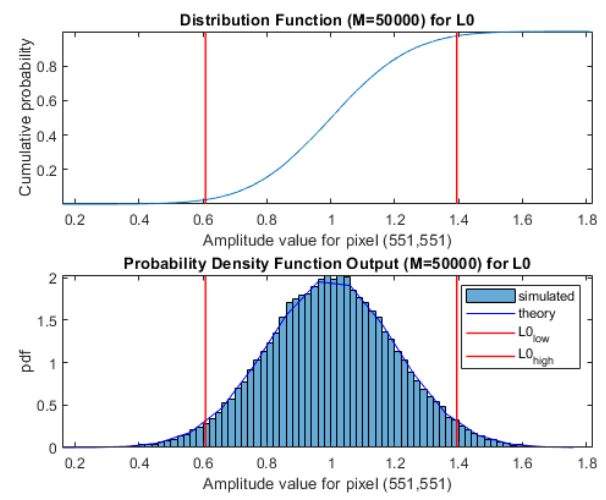

Fig. 4. DF and PDF for amplitude pixel level-0
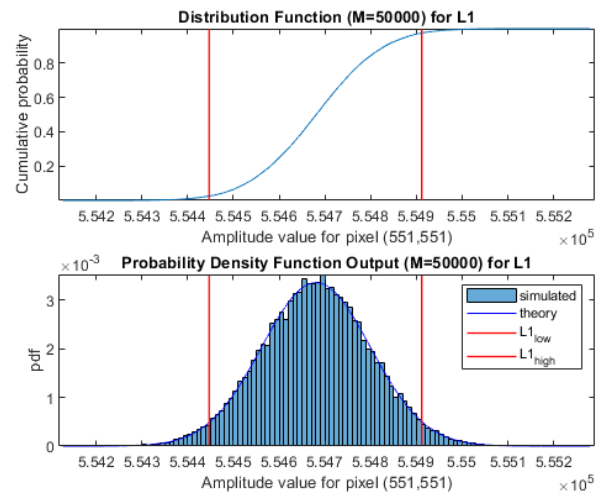

Fig. 5. DF and PDF for amplitude pixel level-1

steps of the processing chain and the extension to them of the concept of traceability to SI.

\section{REFERENCES}

[1] A. Moreira, P. Prats-Iraola, M. Younis, G. Krieger, I. Hajnsek, and K. P. Papathanassiou, "A tutorial on synthetic aperture radar," IEEE Geoscience and Remote Sensing Magazine, vol. 1, no. 1, pp. 6-43, March 2013.

[2] N Fox, "Qa4eo guide: Qa4eo-qaeo-gen-dqk-001: a guide to establish a quality indicator on a satellite sensor derived data product (version 4.0)," See http://qa4eo. org/docs/QA4EO-QAEOGEN-DQK-001_v4. 0. pdf, 2010.

[3] BIPM, IEC, IFCC, ILAC, ISO, IUPAC, IUPAP, and OIML, "Evaluation of measurement data - guide to the expression of uncertainty in measurement, JCGM 100: 2008 GUM 1995 with minor corrections," Joint Committee for Guides in Metrology, September 2008.

[4] A. Pearlman, R. Datla, R. Kacker, and C. Cao, "Translating radiometric requirements for satellite sensors to match international standards," Journal of research of the National Institute of Standards and Technology, vol. 119, pp. 272, 2014.

[5] J. Gorroño, F. Gascon, and N. P Fox, "Radiometric uncertainty per pixel for the sentinel-2 11c products," in SPIE Remote Sensing. International Society for Optics and Photonics, 2015, pp. 96391G-96391G.

[6] I. G. Cumming and F. H. Wong, Digital processing of synthetic aperture radar data: algorithms and implementation, Artech house, 2005.

[7] G. Franceschetti, R. Guida, A. Iodice, D. Riccio, and G. Ruello, "Efficient simulation of hybrid stripmap/spotlight sar raw signals from extended scenes," IEEE Transactions on Geoscience and Remote Sensing, vol. 42, no. 11, pp. 2385-2396, 2004.

[8] P. Howarth and F. Redgrave, Metrology - in short, Third edition, 2008.

[9] BIPM, IEC, IFCC, ILAC, ISO, IUPAC, IUPAP, and OIML, "Evaluation of measurement data - supplement 1 to the 'guide to the expression of uncertainty in measurement' - propagation of distributions using a monte carlo method, JCGM 101: 2008," Joint Committee for Guides in Metrology, 2008. 